Póster

Caracterización del hirsutismo en una población de mujeres atendidas en consulta externa de ginecología en una IPS de la ciudad de Manizales

\author{
González $L J^{1,2}$, Aguirre $V E^{1}$, Giraldo $J F^{1}$.
}

Universidad de Manizales ${ }^{1}$, Manizales, Colombia, Centro de Fertilidad y Endocrinología Reproductiva CEFERTIL SAS', Manizales, Colombia.

Objetivo: Caracterizar un grupo de pacientes hirsutas identificadas en la ciudad de Manizales, Caldas, para determinar la prevalencia de sus causas.

- Caracterizar población hirsuta en consulta especializada.

- Determinar factores asociados al hirsutismo.

- Determinar en la población estudiada los principales riesgos asociados a trastornos cardiometabólicos.

Se plantean las siguientes hipótesis nulas y alternativas que se pretenden demostrar:

H0: El hirsutismo y el IMC son variables independientes.

H1: Existe asociación entre la presencia de hirsutismo y el IMC.

H0: El hirsutismo y el antecedente personal de otras endocrinopatías son variables independientes.

H1: Existe asociación entre la presencia de hirsutismo y el antecedente personal de endocrinopatías.

H0: El hirsutismo y el antecedente de acné son variables independientes.

H1: Existe asociación entre la presencia de hirsutismo y el antecedente de acné.

H0: El hirsutismo y el antecedente de trastornos del metabolismo de los carbohidratos son variables independientes.

H1: Existe asociación entre la presencia de hirsutismo y el antecedente de trastornos del metabolismo de los carbohidratos.

H0: El hirsutismo y el antecedente de infertilidad son variables independientes.

H1: Existe asociación entre la presencia de hirsutismo y el antecedente de infertilidad.

H0: El hirsutismo y el antecedente de hiperprolactinemia son variables independientes.

H1: Existe asociación entre la presencia de hirsutismo y el antecedente de hiperprolactinemia.

H0: El hirsutismo y la acantosis nigricans son variables independientes.

H1: Existe asociación entre la presencia de hirsutismo y el antecedente de acantosis nigricans.

H0: La distribución de las causas de hirsutismo en la población de estudio es igual a la referida en estudios norteamericanos y europeos.

H1: La distribución de hirsutismo en la población de estudio es diferente a la referida en estudios norteamericanos y europeos.
Introducción: El hirsutismo en la mujer se define como crecimiento del pelo terminal en áreas andrógeno-sensibles, de normal crecimiento en el varón. El $80 \%$ de las veces este signo clínico en las mujeres es producido por el síndrome de ovario poliquístico (SOP). Definido éste como un factor de riesgo cardiometabólico y reproductivo. Podemos inferir entonces que 8 de cada 10 mujeres hirsutas tienen 7 veces más probabilidades de sufrir diabetes mellitus, un $40 \%$ de riesgo incrementado de intolerancia a los carbohidratos y un 30 a $50 \%$ de posibilidades de tener resistencia a la insulina, cuando esta patología se asocia con obesidad los riesgos cardiometabólicos son aún más marcados. Si la paciente cursa con embarazo tiene mayor riesgo de preclamsia, diabetes gestacional y restricción del crecimiento fetal.

La determinación de la presencia de hirsutismo se basa especialmente en una exploración clínica, es por esto que el grupo de investigación considera importante la revisión de una cohorte que confirme que la presencia de hirsutismo debe ser considerado igualmente como indicador de riesgo cardiometabólico y reproductivo, así como de síndrome de ovario poliquístico. La escala de graduación utilizada es la de Ferriman Gallwey, consignada en un instrumento de fácil aplicación por el personal de salud, el médico general y el especialista, no ha sido aún ampliamente difundida, y este es uno de los propósitos de este estudio.

Entre los criterios para diagnosticar síndrome de ovario poliquístico establecido por la sociedad de exceso de andrógenos, consenso 2006, determina que deben descartarse otras causas de hirsutismo, como hiperplasia suprarrenal congénita de aparición tardía, hiperprolactinemia, disfunción tiroidea, síndrome de Cushing, tumores productores de andrógenos. Nuestro objetivo en esta caracterización es determinar la proporción de las diferentes causas del mismo. Existen límites no claros entre algunas de estas causas como por ejemplo la condición de hiperprolactinemia como causa o resultado de un estado hiperandrogénico, la asociación entre síndrome de ovario poliquístico y la presencia de hipotiroidismo, este último determinado como criterio de exclusión para SOP, creemos entonces que en algunos casos puede haber superposición de causas que no descartan de manera absoluta la presencia de esta patología.

Planteamiento del problema y justificación: El hirsutismo, como indicador de la presencia de posible diagnóstico del síndrome de ovario poliquístico, se puede considerar un buen indicador de factor de riesgo. Ahora bien, detectar oportunamente una paciente con esta patología y manejarla de forma adecuada disminuye la probabilidad de la aparición de patologías cardiometabólicas graves como diabetes mellitus tipo 2, síndrome metabólico, resistencia periférica a la insulina, entre otras. Este estudio pretende que con la aplicación en la consulta externa de un instrumento sencillo como es la escala de Ferriman Gallwey se identifiquen de forma temprana y oportuna aquellas pacientes con hirsutismo como marcador biológico del desarrollo del síndrome de ovario poliquístico.

Metodología: Se trata de un estudio descriptivo, poblacional. Consiste en la revisión de historias clínicas de las pacientes atendidas en la consulta externa especializada de atención en la ciudad de Manizales. La finalidad es realizar un análisis y una 
descripción de lo encontrado para dar respuesta a las hipótesis planteadas aun en discusión en la literatura y afectadas por características demográficas independientes en cada región.

Conclusiones: El SOP, causa principal asociada a hirsutismo en su presentación, frecuencia y complicaciones está influenciado por factores medioambientales y demográficos, es la razón por la cual los alcances de la revisión de esta cohorte son fundamentales para nuestra población y punto de partida para estudios futuros en el tema, en búsqueda de determinar de esta forma estrategias de la detección temprana de factores de riesgo asociados a estados hiperandrogénicos, partiendo de la simple visualización de la distribución del pelo terminal.
Póster

La frecuencia de consumo de verduras y frutas en los niños con sobrepeso, en relación con sus padres

\section{Awadalla Shokery}

Hospital San José, Bogotá, Colombia.

La obesidad se relaciona con un mayor consumo de comida rápida y procesada y disminución del consumo de frutas y verduras. Para evaluar los hábitos alimentarios de los niños en relación con sus padres, se diseñó un cuestionario para el registro de estos hábitos y los datos antropométricos.

En total, 422 niños con sobrepeso fueron incluidos (250 niños, 172 niñas); la edad de los niños fue de $13 \pm 0,9$ años (M \pm DS). El Indice de masa corporal (IMC) fue $\supseteq$ de P85. Se excluyeron las anormalidades hormonales y genéticos. Ninguno de los niños consumía las cinco porciones diarias recomendadas de frutas y verduras. De 210 niños (110 niñas, 100 niños), 55\% no consumen frutas ni verduras, los padres no les ofrecen, ya que no les gusta; sólo llevan una fruta para las onces del colegio y generalmente no se la comen. Los padres de este grupo sólo toman jugo en la mañana. De 82 niños, 19\% (50 niñas, 32 niños) comen fruta sólo en el desayuno. Los padres de este grupo comen frutas y verduras dos veces al día. 130 niños (30\%) tienen una fruta en el desayuno y algunas verduras en la cena, sus padres consumen de tres a cuatro porciones de frutas y verduras. Los padres consideran el jugo como consumo de fruta.

En conclusión, los niños con sobrepeso no consumen suficientes frutas y verduras. Hay una fuerte correlación entre los hábitos de los padres y lo de los niños. Fomentar el consumo de frutas y verduras debe ser parte de la prevención de la obesidad en los niños al igual que en sus padres.
Póster

Causas del fracaso de programas de reducción de peso en niños

Awadalla Shokery

Hospital San Jose, Bogotá, Colombia.

Aunque parezca fácil el hecho de comer sano y hacer más ejercicio para el manejo de la obesidad, la reducción de peso en los niños es una tarea difícil. Para aclarar las principales causas de este fracaso se incluyen 200 familias con uno o más niños obesos. En total 235, 122 niños y 113 niñas con índice de masa corporal > percentil 95 fueron incluidos. La edad fue de $11 \pm 0,9$ años (M $\pm D S$ ). Se excluyeron los niños con obesidad de causas genéticas y hormonales. Todas las familias fueron instruidas sobre las recomendaciones y los niños fueron evaluados cada dos meses durante seis meses. Cuestionarios sobre el cumplimiento de las recomendaciones se realizaron en cada visita. Los primeros dos meses, 80 niños (65\%) y 90 niñas $(79 \%)$ bajaron de peso. Los segundos dos meses, este número se ha reducido a 60 niños (49\%) y 72 niñas (63\%). Los siguientes dos meses, sólo 45 niños (36\%) y 60 niñas (53\%) bajaron de peso. La falta de tiempo de los padres para hacer ejercicio fue la razón principal para el fracaso en la pérdida de peso (55\%). La segunda causa fue la no cooperación de los abuelos, al incumplir las recomendaciones alimentarias $(32 \%)$. Otras causas (18\%) fueron la negativa de los niños para consumir los alimentos ofrecidos y hacer ejercicio. En conclusiones: las causas del fracaso de los programas de perder peso en los niños, en la mayoría de los casos, es responsabilidad de los padres y abuelos (87\%). Los programas de educación deben ser dirigidos a los padres y a los abuelos para tener mayor adherencia a las recomendaciones. 